Proyecciones Journal of Mathematics

Vol. 35, $\mathrm{N}^{o}$ 4, pp. 481-490, December 2016.

Universidad Católica del Norte

Antofagasta - Chile

\title{
Some geometric properties of lacunary Zweier Sequence Spaces of order $\alpha$
}

\author{
Karan Tamang \\ North Eastern Regional Institute of Science and Tech., India \\ and \\ Bipan Hazarika \\ Rajiv Gandhi University, India \\ Received: July 2016. Accepted : September 2016
}

\begin{abstract}
In this paper we introduce a new sequence space using Zweier matrix operator and lacunary sequence of order $\alpha$. Also we study some geometrical properties such as order continuous, the Fatou property and the Banach-Saks property of the new space.
\end{abstract}

Keywords and phrases : Lacunary sequence; Zweier operator; order continuous; Fatou property; Banach-Saks property

AMS subject classification (2010) : 40A05, 40D25, $46 A 45$. 


\section{Introduction}

Throughout the article $w, c, c_{0}$ and $\ell_{\infty}$ denotes the spaces of all, convergent, null and bounded sequences, respectively. Also, by $\ell_{1}$ and $\ell_{p}$, we denote the spaces of all absolutely summable and $p$-absolutely summable series, respectively. Recall that a sequence $(x(i))_{i=1}^{\infty}$ in a Banach space $X$ is called Schauder (or basis) of $X$ if for each $x \in X$ there exists a unique sequence $(a(i))_{i=1}^{\infty}$ of scalars such that $x=\sum_{i=1}^{\infty} a(i) x(i)$, i.e. $\lim _{n \rightarrow \infty} \sum_{i=1}^{n} a(i) x(i)=$ $x$. A sequence space $X$ with a linear topology is called a $K$-space if each of the projection maps $P_{i}: X \rightarrow \mathbf{C}$ defined by $P_{i}(x)=x(i)$ for $x=(x(i))_{i=1}^{\infty} \in$ $X$ is continuous for each natural $i$. A Fréchet space is a complete metric linear space and the metric is generated by a $F$-norm and a Fréchet space which is a $K$-space is called an $F K$-space i.e. a $K$-space $X$ is called an FKspace if $X$ is a complete linear metric space. In other words, $X$ is an $F K$ space if $X$ is a Fréchet space with continuous coordinatewise projections. All the sequence spaces mentioned above are $F K$-space except the space $c_{00}$ which is the space of real sequences which have only a finite number of nonzero coordinates. An $F K$-space $X$ which contains the space $c_{00}$ is said to have the property $A K$ if for every sequence $(x(i))_{i=1}^{\infty} \in X, x=\sum_{i=1}^{\infty} x(i) e(i)$ where $e(i)=\left(0,0, \ldots 1^{i^{t h}}\right.$ place $\left., 0,0, \ldots\right)$.

A Banach space $X$ is said to be a Köthe sequence space if $X$ is a subspace of $w$ such that

(a) if $x \in w, y \in X$ and $|x(i)| \leq|y(i)|$ for all $i \in \mathbf{N}$, then $x \in X$ and $\|x\| \leq\|y\|$

(b) there exists an element $x \in X$ such that $x(i)>0$ for all $i \in \mathbf{N}$.

We say that $x \in X$ is order continuous if for any sequence $\left(x_{n}\right) \in X$ such that $x_{n}(i) \leq|x(i)|$ for all $i \in \mathbf{N}$ and $x_{n}(i) \rightarrow 0$ as $n \rightarrow \infty$ we have $\left\|x_{n}\right\| \rightarrow 0$ holds.

A Köthe sequence space $X$ is said to be order continuous if all sequences in $X$ are order continuous. It is easy to see that $x \in X$ order continuous if and only if $\|(0,0, \ldots, 0, x(n+1), x(n+2), \ldots)\| \rightarrow 0$ as $n \rightarrow \infty$.

A Köthe sequence space $X$ is said to be the Fatou property if for any real sequence $x$ and $\left(x_{n}\right)$ in $X$ such that $x_{n} \uparrow x$ coordinatewisely and $\sup _{n}\left\|x_{n}\right\|<\infty$, we have that $x \in X$ and $\left\|x_{n}\right\| \rightarrow\|x\|$. 
A Banach space $X$ is said to have the Banach-Saks property if every bounded sequence $\left(x_{n}\right)$ in $X$ admits a subsequence $\left(z_{n}\right)$ such that the sequence $\left(t_{k}(z)\right)$ is convergent in $X$ with respect to the norm, where

$$
t_{k}(z)=\frac{z_{1}+z_{2}+\ldots+z_{k}}{k} \text { for all } k \in \mathbf{N} .
$$

Some of works on geometric properties of sequence space can be found in $[3,4,8,9,13,16,17,18,19,20,22,23]$.

Şengönül [24] defined the sequence $y=\left(y_{k}\right)$ which is frequently used as the $Z^{i}$-transformation of the sequence $x=\left(x_{k}\right)$ i.e.

$$
y_{k}=i x_{k}+(1-i) x_{k-1}
$$

where $x_{-1}=0, k \neq 0,1<k<\infty$ and $Z^{i}$ denotes the matrix $Z^{i}=\left(z_{n k}\right)$ defined by

$$
z_{n k}=\left\{\begin{array}{cl}
i, & \text { if } n=k \\
1-i, & \text { if } n-1=k \\
0, & \text { otherwise }
\end{array}\right.
$$

Şengönül [24] introduced the Zweier sequence spaces $\mathcal{Z}$ and $\mathcal{Z}_{0}$ as follows

$$
\mathcal{Z}=\left\{x=\left(x_{k}\right) \in w: Z^{i} x \in c\right\}
$$

and

$$
\mathcal{Z}_{0}=\left\{x=\left(x_{k}\right) \in w: Z^{i} x \in c_{0}\right\} .
$$

For details on Zweier sequence spaces we refer to $[5,10,11,12,14,15]$.

\section{Lacunary Zweier sequence spaces of order $\alpha$}

by lacunary sequence we mean an increasing sequence $\theta=\left(k_{r}\right)$ of positive integers satisfyling $k_{0}=0$ and $h_{r}:=k_{r}-k_{r-1} \rightarrow \infty$ as $r \rightarrow \infty$. We denote the intervals, by $I_{r}=\left(k_{r-1}, k_{r}\right]$, which determines $\theta$. Let $\alpha \in(0,1]$ be any real number and let $p$ be a positive real number such that $1 \leq p<\infty$. Now we define the following sequence space.

$$
\left[\mathcal{Z}_{\theta}^{\alpha}\right]_{\infty}(p)=\left\{x \in w: \sup _{r} \frac{1}{h_{r}^{\alpha}} \sum_{k \in I_{r}}\left|\left(Z^{i} x\right)_{k}\right|^{p}<\infty\right\} .
$$

Special cases: 
(a) For $p=1$ we have $\left[\mathcal{Z}_{\theta}^{\alpha}\right]_{\infty}(p)=\left[\mathcal{Z}_{\theta}^{\alpha}\right]_{\infty}$.

(b) For $\alpha=1$ and $p=1$ we have $\left[\mathcal{Z}_{\theta}^{\alpha}\right]_{\infty}(p)=\left[\mathcal{Z}_{\theta}\right]_{\infty}$.

For details on sequence spaces of order $\alpha$ we refer to $[1,2,6,7]$.

Theorem 2.1. Let $\alpha \in(0,1]$ and $p$ be a positive real number such that $1 \leq p<\infty$. Then the sequence space $\left[\mathcal{Z}_{\theta}^{\alpha}\right]_{\infty}(p)$ is a BK-space normed by

$$
\|x\|_{\alpha}=\sup _{r} \frac{1}{h_{r}^{\alpha}}\left(\sum_{k \in I_{r}}\left|\left(Z^{i} x\right)_{k}\right|^{p}\right)^{\frac{1}{p}} .
$$

Proof. Since the matrix $Z^{i}$ is a triangle, we have the result by norm (2.1) and the Theorem 4.3 .12 of Wilansky [[25], p. 63]. $\square\left[\mathcal{Z}_{\theta}^{\alpha}\right]_{\infty} \subset\left[\mathcal{Z}_{\theta}^{\alpha}\right]_{\infty}(p)$.

Theorem 2.2. Let $\alpha$ and $\beta$ be fixed real numbers such that $0<\alpha \leq \beta \leq 1$ and $p$ be a positive real number such that $1 \leq p<\infty$. Then $\left[\mathcal{Z}_{\theta}^{\alpha}\right]_{\infty}(p) \subset$ $\left[\mathcal{Z}_{\theta}^{\beta}\right]_{\infty}(p)$.

Proof. The proof of theorem follows from the following inequality. For all $r \in \mathbf{N}$ we have is straightforward, so omitted.

$$
\frac{1}{h_{r}^{\alpha}} \sum_{k \in I_{r}}\left|\left(Z^{i} x\right)_{k}\right|^{p} \leq \frac{1}{h_{r}^{\beta}} \sum_{k \in I_{r}}\left|\left(Z^{i} x\right)_{k}\right|^{p}
$$

Theorem 2.3. Let $\alpha$ and $\beta$ be fixed real numbers such that $0<\alpha \leq \beta \leq 1$ and $p$ be a positive real number such that $1 \leq p<\infty$. For two lacunary sequences $\theta=\left(h_{r}\right)$ and $\phi=\left(l_{r}\right)$ for all $r$, then $\left[\mathcal{Z}_{\theta}^{\alpha}\right]_{\infty}(p) \subset\left[\mathcal{Z}_{\phi}^{\beta}\right]_{\infty}(p)$ if and only if $\sup _{r}\left(\frac{h_{r}^{\alpha}}{l_{r}^{\beta}}\right)<\infty$.

Proof. Let $x=\left(x_{k}\right) \in\left[\mathcal{Z}_{\theta}^{\alpha}\right]_{\infty}(p)$ and $\sup _{r}\left(\frac{h_{r}^{\alpha}}{l_{r}^{\beta}}\right)<\infty$. Then

$$
\sup _{r} \frac{1}{h_{r}^{\alpha}} \sum_{k \in I_{r}}\left|\left(Z^{i} x\right)_{k}\right|^{p}<\infty
$$


and there exists a positive number $K$ such that $h_{r}^{\alpha} \leq K l_{r}^{\beta}$ and so that $\frac{1}{l_{r}^{\beta}} \leq \frac{K}{h_{r}^{\alpha}}$ for all $r$. Therefore, we have

$$
\frac{1}{l_{r}^{\beta}} \sum_{k \in I_{r}}\left|\left(Z^{i} x\right)_{k}\right|^{p} \leq \frac{K}{h_{r}^{\alpha}} \sum_{k \in I_{r}}\left|\left(Z^{i} x\right)_{k}\right|^{p} .
$$

Now taking supremum over $r$, we get

$$
\sup _{r} \frac{1}{l_{r}^{\beta}} \sum_{k \in I_{r}}\left|\left(Z^{i} x\right)_{k}\right|^{p} \leq \sup _{r} \frac{K}{h_{r}^{\alpha}} \sum_{k \in I_{r}}\left|\left(Z^{i} x\right)_{k}\right|^{p}
$$

and hence $x \in\left[\mathcal{Z}_{\phi}^{\beta}\right]_{\infty}(p)$.

Next suppose that $\left[\mathcal{Z}_{\theta}^{\alpha}\right]_{\infty}(p) \subset\left[\mathcal{Z}_{\phi}^{\beta}\right]_{\infty}(p)$ and $\sup _{r}\left(\frac{h_{r}^{\alpha}}{l_{r}^{\beta}}\right)=\infty$. Then there exists an incresing sequence $\left(r_{i}\right)$ of natural numbers such that $\lim _{i}\left(\frac{h_{r_{i}}^{\alpha}}{l_{r_{i}}^{\beta}}\right)=\infty$. Let $L$ be a positive real number, then there exists $i_{0} \in \mathbf{N}$ such that $\frac{h_{r_{i}}^{\alpha}}{l_{r_{i}}^{\beta}}>L$ for all $r_{i} \geq i_{0}$. Then $h_{r_{i}}^{\alpha}>L l_{r_{i}}^{\beta}$ and so $\frac{1}{l_{r_{i}}^{\beta}}>\frac{L}{h_{r_{i}}^{\alpha}}$. Therefore we can write

$$
\frac{1}{l_{r_{i}}^{\beta}} \sum_{k \in I_{r}}\left|\left(Z^{i} x\right)_{k}\right|^{p}>\frac{L}{h_{r_{i}}^{\alpha}} \sum_{k \in I_{r}}\left|\left(Z^{i} x\right)_{k}\right|^{p} \text { for all } r_{i} \geq i_{0} .
$$

Now taking supremum over $r_{i} \geq i_{0}$ then we get

$$
\sup _{r_{i} \geq i_{0}} \frac{1}{l_{r_{i}}^{\beta}} \sum_{k \in I_{r_{i}}}\left|\left(Z^{i} x\right)_{k}\right|^{p}>\sup _{r_{i} \geq i_{0}} \frac{L}{h_{r_{i}}^{\alpha}} \sum_{k \in I_{r_{i}}}\left|\left(Z^{i} x\right)_{k}\right|^{p} .
$$

Since the relation (2.2) holds for all $L \in \mathbf{R}^{+}$(we may take the number $L$ sufficienlty large), we have

$$
\sup _{r_{i} \geq i_{0}} \frac{1}{l_{r_{i}}^{\beta}} \sum_{k \in I_{r_{i}}}\left|\left(Z^{i} x\right)_{k}\right|^{p}=\infty
$$

but $x=\left(x_{k}\right) \in\left[\mathcal{Z}_{\theta}^{\alpha}\right]_{\infty}(p)$ with

$$
\sup _{r}\left(\frac{h_{r}^{\alpha}}{l_{r}^{\beta}}\right)<\infty .
$$

Therefore $x \notin\left[\mathcal{Z}_{\phi}^{\beta}\right]_{\infty}(p)$ which contradicts that $\left[\mathcal{Z}_{\theta}^{\alpha}\right]_{\infty}(p) \subset\left[\mathcal{Z}_{\phi}^{\beta}\right]_{\infty}(p)$. Hence $\sup _{r \geq 1}\left(\frac{h_{r}^{\alpha}}{l_{r}^{\beta}}\right)<\infty$. 
Corollary 2.4. Let $\alpha$ and $\beta$ be fixed real numbers such that $0<\alpha \leq \beta \leq 1$ and $p$ be a positive real number such that $1 \leq p<\infty$. For any two lacunary sequences $\theta=\left(h_{r}\right)$ and $\phi=\left(l_{r}\right)$ for all $r \geq 1$, then

(a) $\left[\mathcal{Z}_{\theta}^{\alpha}\right]_{\infty}(p)=\left[\mathcal{Z}_{\phi}^{\beta}\right]_{\infty}(p)$ if and only if $0<\inf _{r}\left(\frac{h_{r}^{\alpha}}{l_{r}^{\beta}}\right)<\sup _{r}\left(\frac{h_{r}^{\alpha}}{l_{r}^{\beta}}\right)<\infty$.

(b) $\left[\mathcal{Z}_{\theta}^{\alpha}\right]_{\infty}(p)=\left[\mathcal{Z}_{\phi}^{\alpha}\right]_{\infty}(p)$ if and only if $0<\inf _{r}\left(\frac{h_{r}^{\alpha}}{l_{r}^{\alpha}}\right)<\sup _{r}\left(\frac{h_{r}^{\alpha}}{l_{r}^{\alpha}}\right)<\infty$.

(c) $\left[\mathcal{Z}_{\theta}^{\alpha}\right]_{\infty}(p)=\left[\mathcal{Z}_{\theta}^{\beta}\right]_{\infty}(p)$ if and only if $0<\inf _{r}\left(\frac{h_{r}^{\alpha}}{h_{r}^{\beta}}\right)<\sup _{r}\left(\frac{h_{r}^{\alpha}}{h_{r}^{\beta}}\right)<\infty$.

Theorem 2.5. $\ell_{p} \subset\left[\mathcal{Z}_{\theta}^{\alpha}\right]_{\infty}(p) \subset \ell_{\infty}$.

Proof. The proof of the result is straightforward, so omitted.

Theorem 2.6. If $0<p<q$, then $\left[\mathcal{Z}_{\theta}^{\alpha}\right]_{\infty}(p) \subset\left[\mathcal{Z}_{\theta}^{\alpha}\right]_{\infty}(q)$.

Proof. The proof of the result is straightforward, so omitted.

\section{Some geometric properties}

In this section we study some of the geometric properties like order continuous, the Fatou property and the Banach-Saks property in this new sequence space.

Theorem 3.1. The space $\left[\mathcal{Z}_{\theta}^{\alpha}\right]_{\infty}(p)$ is order continuous.

Proof. We have to show that the space $\left[\mathcal{Z}_{\theta}^{\alpha}\right]_{\infty}(p)$ is an $A K$-space. It is easy to see that $\left[\mathcal{Z}_{\theta}^{\alpha}\right]_{\infty}(p)$ contains $c_{00}$ which is the space of real sequences which have only a finite number of non-zero coordinates. By using the definition of $A K$-properties, we have that $x=(x(i)) \in\left[\mathcal{Z}_{\theta}^{\alpha}\right]_{\infty}(p)$ has a unique representation $x=\sum_{i=1}^{\infty} x(i) e(i)$ i.e. $\left\|x-x^{[j]}\right\|_{\alpha}=\|(0,0, . ., x(j), x(j+$ $1), \ldots) \|_{\alpha} \rightarrow 0$ as $j \rightarrow \infty$, which means that $\left[\mathcal{Z}_{\theta}^{\alpha}\right]_{\infty}(p)$ has $A K$. Therefore $B K$-space $\left[\mathcal{Z}_{\theta}^{\alpha}\right]_{\infty}(p)$ containing $c_{00}$ has $A K$-property, hence the space $\left[\mathcal{Z}_{\theta}^{\alpha}\right]_{\infty}(p)$ is order continuous.

Theorem 3.2. The space $\left[\mathcal{Z}_{\theta}^{\alpha}\right]_{\infty}(p)$ has the Fatou property. 
Proof. Let $x$ be a real sequence and $\left(x_{j}\right)$ be any nondecreasing sequence of non-negative elements form $\left[\mathcal{Z}_{\theta}^{\alpha}\right]_{\infty}(p)$ such that $x_{j}(i) \rightarrow x(i)$ as $j \rightarrow \infty$ coordinatewisely and $\sup _{j}\left\|x_{j}\right\|_{\alpha}<\infty$.

Let us denote $T=\sup _{j}\left\|x_{j}\right\|_{\alpha}$. Since the supremum is homogeneous, then we have

$$
\begin{gathered}
\frac{1}{T} \sup _{r} \frac{1}{h_{r}^{\alpha}}\left(\sum_{k \in I_{r}}\left|\left(Z^{i} x_{j}(i)\right)_{k}\right|^{p}\right)^{\frac{1}{p}} \\
\leq \sup _{r} \frac{1}{h_{r}^{\alpha}}\left(\sum_{k \in I_{r}}\left|\frac{\left(Z^{i} x_{j}(i)\right)_{k}}{\left\|x_{n}\right\|_{\alpha}}\right|^{p}\right)^{\frac{1}{p}} \\
=\frac{1}{\left\|x_{n}\right\|_{\alpha}}\left\|x_{n}\right\|_{\alpha}=1 .
\end{gathered}
$$

Also by the assumptions that $\left(x_{j}\right)$ is non-dreceasing and convergent to $x$ coordinatewisely and by the Beppo-Levi theorem, we have

$$
\begin{aligned}
& \frac{1}{T} \lim _{j \rightarrow \infty} \sup _{r} \frac{1}{h_{r}^{\alpha}}\left(\sum_{k \in I_{r}}\left|\left(Z^{i} x_{j}(i)\right)_{k}\right|^{p}\right)^{\frac{1}{p}} \\
& =\sup _{r} \frac{1}{h_{r}^{\alpha}}\left(\sum_{k \in I_{r}}\left|\frac{\left(Z^{i} x(i)\right)_{k}}{T}\right|^{p}\right)^{\frac{1}{p}} \leq 1
\end{aligned}
$$

whence

$$
\|x\|_{\alpha} \leq T=\sup _{j}\left\|x_{j}\right\|_{\alpha}=\lim _{j \rightarrow \infty}\left\|x_{j}\right\|_{\alpha}<\infty .
$$

Therefore $x \in\left[\mathcal{Z}_{\theta}^{\alpha}\right]_{\infty}(p)$. On the other hand, since $0 \leq x$ for any natural number $j$ and the sequence $\left(x_{j}\right)$ is non-decreasing, we obtain that the sequence $\left(\left\|x_{j}\right\|_{\alpha}\right)$ is bounded form above by $\|x\|_{\alpha}$. Therefore $\lim _{j \rightarrow \infty}\left\|x_{j}\right\|_{\alpha} \leq$ $\|x\|_{\alpha}$ which contadicts the above inequality proved already, yields that $\|x\|_{\alpha}=\lim _{j \rightarrow \infty}\left\|x_{j}\right\|_{\alpha}$.

Theorem 3.3. The space $\left[\mathcal{Z}_{\theta}^{\alpha}\right]_{\infty}(p)$ has the Banach-Saks property.

Proof. The proof of the result follows from the standard technique. 


\section{References}

[1] R. Çolak, C. A. Bektaş $\lambda$-statistical convergence of order $\alpha$, Acta Math. Sci., 31 (3), pp. 953-959, (2011).

[2] R. Çolak, Statistical Convergence of Order $\alpha$, Modern Methods in Analysis and Its Applications, pp. 121-129. Anamaya Pub., New Delhi (2010).

[3] Y. A. Cui, H. Hudzik, On the Banach-Saks and weak Banach-Saks properties of some Bannach sequence spaces, Acta Sci. Math.(Szeged), 65, pp. 179-187, (1999).

[4] J. Diestel, Sequence and Series in Banach spaces, in Graduate Texts in Math., Vol. 92, Springer-Verlag, (1984).

[5] A. Esi, A. Sapszoğlu, On some lacunary $\sigma$-strong Zweier convergent sequence spaces, Romai J., 8 (2), pp. 61-70, (2012).

[6] M. Et, M. Çinar, M. Karakaş, On $\lambda$-statistical convergence of order $\alpha$ of sequences of function, J. Inequa. Appl., 2013:204, (2013).

[7] M. Et, S. A. Mohiuddine, A. Alotaibi, On $\lambda$-statistical convergence and strongly $\lambda$-summable functions of order $\alpha$, J. Inequa. Appl., 2013:469, (2013).

[8] M. Et, V. Karakaya, A new difference sequence set of order $\alpha$ and its geometrical properties, Abst. Appl. Anal., Volume 2014, Article ID 278907, 4 pages, (2014).

[9] M. Et, M. Karakaş, Muhammed Çinar, Some geometric properties of a new modular space defined by Zweier operator, Fixed point Theory Appl., 2013:165, (2013).

[10] B. Hazarika, K. Tamang, B. K. Singh, Zweier ideal convergent sequence spaces defined by Orlicz function, The J. Math. Comp. Sci., 8 (3), pp. 307-318, (2014).

[11] B. Hazarika, K. Tamang, B. K. Singh, On paranormed Zweier ideal convergent sequence spaces defined by Orlicz Function, J. Egypt. Math. Soc., 22 (3), pp. 413-419, (2014). 
[12] Y. F. Karababa and A. Esi, On some strong Zweier convergent sequence spaces, Acta Univ. Apulensis, 29, pp. 9-15, (2012).

[13] M. Karakaş, M. Et, V. Karakaya, Some geometric properties of a new difference sequence space involving lacunary sequences, Acta Math. Ser. B. Engl. Ed., 33 (6), pp. 1711-1720, (2013).

[14] V. A. Khan, K. Ebadullah, A. Esi, N. Khan, M. Shafiq, On Paranorm Zweier $\mathcal{I}$-convergent sequences spaces, Inter. J. Anal., Vol. 2013, Article ID 613501, 6 pages, (2013).

[15] V. A. Khan, K. Ebadullah, A. Esi, M. Shafiq, On some Zweier Iconvergent sequence spaces defined by a modulus function, Afr. Mat. DOI 10.1007/s13370-013-0186-y (2013).

[16] V. A. Khan, A. H. Saifi, Some geometric properties of a generalized Cesáro Masielak-Orlicz sequence space, Thai J. Math., 1 (2), pp. 97108, (2003).

[17] V. A. Khan, Some geometric properties for Nörlund sequence spaces, Nonlinear Anal. Forum, 11(1), pp. 101-108, (2006).

[18] V. A. Khan, Some geometric properties of a generalized Cesáro sequence space, Acta Math. Univ. Comenian, 79 (1), pp. 1-8, (2010).

[19] V. A. Khan, Some geometrical properties of Riesz-Musielak-Orlicz sequence spaces, Thai J. Math., 8(3), pp. 565-574, (2010).

[20] V. A. Khan, Some geometrical properties of generalized lacunary strongly convergent sequence space, J. Math. Anal., 2(2), pp. 6-14, (2011).

[21] L. Leindler, Über die la Vallée-Pousinsche Summierbarkeit Allgemeiner Orthogonalreihen. Acta Math. Acad. Sci. Hung., 16, pp. 375-387, (1965).

[22] M. Mursaleen, R. Çolak, M. Et, Some geometric inequalities in a new Banach sequence space, J. Ineq. Appl., Article ID 86757, 6, (2007).

[23] M. Mursaleen, V. A. Khan, Some geometric properties of a sequence space of Riesz mean, Thai J. Math., 2, pp. 165-171, (2004).

[24] M. Şengönül, On the Zweier sequence space, Demonstratio Math. Vol.XL No. (1), pp. 181-196, (2007). 
[25] A. Wilansky, Summability Theory and its Applications, North-Holland Mathematics Studies 85, Elsevier Science Publications, Amsterdam, New York: Oxford, (1984).

\section{Karan Tamang}

Department of Mathematics,

North Eastern Regional Institute of Science and Technology,

Nirjuli 791109,

Arunachal Pradesh,

India

e-mail : karanthingh@gmail.com

and

\section{Bipan Hazarika}

Department of Mathematics, Rajiv Gandhi University,

Rono Hills,

Doimukh 791112,

Arunachal Pradesh,

India

e-mail : bh_rgu@yahoo.co.in 